\title{
Targeted Mesoporous Iron Oxide Nanoparticles- Encapsulated Perfluorohexane and a Hydrophobic Drug for Deep Tumor Penetration and Therapy
}

\author{
Yu-Lin Su, Jen-Hung Fang, Chia-Ying Liao, Chein-Ting Lin, Yun-Ting Li, Shang-Hsiu Hu ${ }^{凶}$ \\ Department of Biomedical Engineering and Environmental Sciences, National Tsing Hua University, No. 101, Section 2, Kuang-Fu Road, Hsinchu, \\ Taiwan 30013 \\ $\triangle$ Corresponding author: shhu@mx.nthu.edu.tw
}

(c) 2015 Ivyspring International Publisher. Reproduction is permitted for personal, noncommercial use, provided that the article is in whole, unmodified, and properly cited. See http://ivyspring.com/terms for terms and conditions.

Received: 2015.06.01; Accepted: 2015.07.21; Published: 2015.08.09

\begin{abstract}
A magneto-responsive energy/drug carrier that enhances deep tumor penetration with a porous nano-composite is constructed by using a tumor-targeted lactoferrin (Lf) bio-gate as a cap on mesoporous iron oxide nanoparticles (MIONs). With a large payload of a gas-generated molecule, perfluorohexane (PFH), and a hydrophobic anti-cancer drug, paclitaxel (PTX), Lf-MIONs can simultaneously perform bursting gas generation and on-demand drug release upon high-frequency magnetic field (MF) exposure. Biocompatible PFH was chosen and encapsulated in MIONs due to its favorable phase transition temperature $\left(56^{\circ} \mathrm{C}\right)$ and its hydrophobicity. After a short-duration MF treatment induces heat generation, the local pressure increase via the gasifying of the PFH embedded in MION can substantially rupture the three-dimensional tumor spheroids in vitro as well as enhance drug and carrier penetration. As the MF treatment duration increases, Lf-MIONs entering the tumor spheroids provide an intense heat and burst-like drug release, leading to superior drug delivery and deep tumor thermo-chemo-therapy. With their high efficiency for targeting tumors, Lf-MIONs/PTX-PFH suppressed subcutaneous tumors in 16 days after a single MF exposure. This work presents the first study of using MF-induced PFH gasification as a deep tumor-penetrating agent for drug delivery.
\end{abstract}

Key words: Mesoporous particles, drug delivery, combinatorial therapy, magneto-responsive, 3D tumor model.

\section{Introduction}

Advanced drug delivery nano-systems provide promising and novel approaches for improving the efficacy of existing drugs and cancer therapeutic modalities. Numerous nano-systems have demonstrated the potential to achieve reduced side effects, enhanced therapeutic efficiency, increased circulation times, and on-demand drug release. Some of them have been approved for clinical applications in tumor therapy, such as Doxil (PEGylated liposome), Abraxane (paclitaxel albumin-stabilized nanoparticle), DepoCyt (liposomal cytarabine), Oncaspar (PEG-asparaginase), CAELYX ${ }^{\mathrm{TM}}$ (PEGylated liposomal doxorubicin), and Genexol-PM (polymeric micellar nanoparticle) [1-6].
Others have also been demonstrated to possess on-demand release behaviors, which enable release of their therapeutic cargos without any lag in response to a specific physical stimulus, such as $\mathrm{pH}$, light, temperature, ultrasound, and magnetic field [7-11]. Despite the improved therapeutic efficiency, most of these nanoparticles that are delivered through intravenous injection to a targeted tumor site are usually attached on the cancerous cells of the tumor periphery near blood vessels, thus leading to modest survival benefits, as the physiological barrier of the tumor hinders the penetration of nanoparticles and anticancer drugs into the whole interior [12-14]. This 
barrier that reduces transcapillary transport is mainly attributed to the high tumor interstitial fluid pressure (IFP) which reduces the penetration of small molecular drugs but also causes nanoparticle-based drug delivery systems to release their therapeutic agents in the perivascular cells of tumor [15-18]. Additionally, cancer-associated fibroblasts form a dense fiber network in the tumor that can also block the penetration of drugs [19-21]. Therefore, delivering therapeutic agents to cancer cells residing in the center of tumors is difficult due to the native barriers separating the core of the tumor from blood vessels $[22,23]$. To enhance the delivering efficiency of drugs to deep tumor is a key factor to enhance the tumor therapy.

A simple solution to improve the penetration and delivery capacity of drug carriers is to engineer their physic-chemical properties. For example, surface charges dominate the behaviors of nanoparticles (NPs) in tumor environments. Positively charged NPs increase the delivery of payloads to cells, and negatively charged NPs provide better drug delivery into deep tumors $[24,25]$. In recent years, the improvements in controlling nanoparticle size through colloidal chemistry and nanotechnology have also enabled considerable progress in biomedical applications [26]. Many studies have shown that organic carriers (polymeric micelles) and inorganic NPs (gold and silica NPs) with small sizes $(<100 \mathrm{~nm})$ can increase tumor penetration compared to that for larger sizes. For example, Kumar et al. utilized $5.2 \mathrm{~nm}$ gold NPs stabilized with targeting peptides to enhance cellular uptake, whereas Wong and co-workers increased the penetration using a multistage delivery system (up to $100 \mathrm{~nm}$, quantum dot (QD)-coated gelatin spheres) whose sizes could decrease due to collagen degradation at the tumor site, subsequently enabling penetration deep into the tumor [27-29]. However, the tumor penetration capability depends on the properties of ultra-small-sized NPs themselves, and these small NPs cannot carry large amounts of drugs alone. Thus, it is highly desirable to use large NPs (> $100 \mathrm{~nm})$ that can pass through physiological barriers in solid tumors for deep penetration.

Despite these recent advances in drug carrier engineering, technological challenges in effectively delivering nano-platforms with large amounts of therapeutic compounds to tumors still exist. First, it is often difficult to decrease the tumor IFP due to the dense cancer cells around tumor, which blocks the drug carriers outside. In this regard, a popular approach is the application of energy such as hyperthermia and ultrasound, which has been demonstrated to increase tumor blood flow and tumor mi- cro-environmental permeability [30-32]. For example, by combining thermal and mechanical effects, Lai reported that the accumulation of liposomes increased up to threefold to as much as $22 \% \mathrm{ID} / \mathrm{g}$ under the ultrasound and hyperthermia treatment [32,33]. However, such high energy is difficult to control its working area, probably damaging the normal tissue. The second technological difficulty stems from obtaining sufficient drug accumulation at the tumor site, i.e., the kinetics of drug release. In most drug delivery systems, drug release is regulated by the diffusion rate of drugs or the degradation rate of the carrier matrix; these factors limit the achievable local drug concentration. However, practically all therapeutic agents possess a limited therapeutic window (too little is insufficient to kill the cancer cells). Therefore, precise temporal- and dosage-control drug release at deep tumor sites is another key factor for cancer therapy.

In this context, as schematically illustrated in Figure 1a, we report a new class of protein-capped magnetic mesoporous particles with remotely controlled perfluorohexane (PFH) gasifying and hydrophobic anti-cancer drug release for deep tumor penetration and therapy. The protein, lactoferrin (Lf), has a targeting function, forms the shell, and minimizes unintended cargo release; the mesoporous iron oxide nanoparticle (MION) carries a large payload of cargo in the hydrophobic pores and also serves as the actuator for triggering drug release. The hydrophobic paclitaxel (PTX) and perfluorohexane (PFH) that are sequentially encapsulated in Lf-MIONs undergo minimal release until a high-frequency magnetic field (MF) triggers the release locally. Furthermore, the drug delivery system was tracked in a three-dimensional tumor spheroid model to evaluate the particle and drug penetration in real time. Enhanced delivery of MIONs to tumor spheroids can be achieved by Lf targeting and PFH assistance. After a short-time MF exposure, the gasified PFH is actuated by the local temperature increase of MION and thus ruptures and damages the tumor spheroids, leading to a high penetration efficiency of the delivery system. As the duration of MF treatment increases, the synergistic effect of the chemo- and thermo-therapy in the tumor spheroids can lead to the eradication of cells in a deep tumor (Figure 1b). Integrating these functionalities in Lf-MIONs makes it possible to have a potentially practical delivery platform for controlled drug release, bioimaging, disease theragonosis and combination therapy through both multi-cargo delivery and magnetothermal therapy. 
(a)

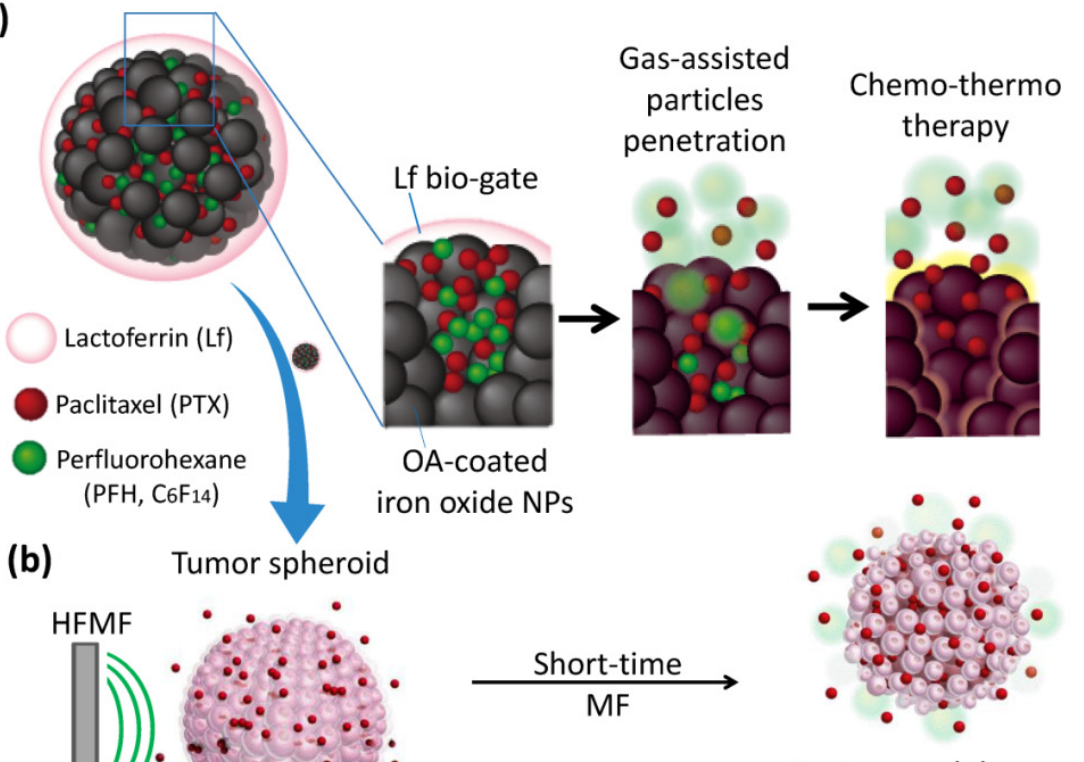

Rupture and damage tumor spheroid

Live cell

Dead cell
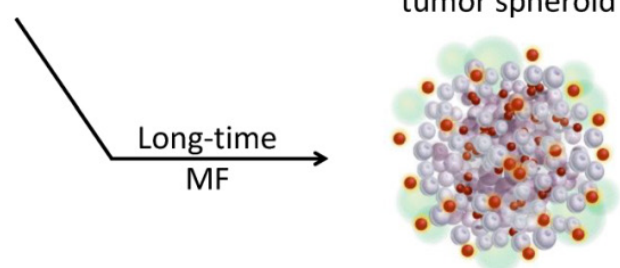

Chemo-thermo

therapy

Figure 1. (a) Schematic illustration of the lactoferrin-capped mesoporous iron oxide nanoparticle (Lf-MION). The hydrophobic pores of Lf-MION carry both paclitaxel (PTX) and perfluorohexane (PFH), and these cargos can be triggered when treated with a high-frequency magnetic field (MF). (b) MF-induced heat gasifies PFH to enhance the penetration and accumulation of Lf-MIONs in a tumor spheroid. Through a combination of thermo-gasification and thermo-chemo-therapy, the Lf-MIONs can effectively eradicate the cancer cells in tumor spheroids.

\section{Materials and Methods}

\section{Synthesis of mesoporous iron oxide nanopar- ticles (MIONs)}

MIONs were prepared via a ligand-assisted method by the introduction of oleic amine (OA, Riedel-de Haën) and sodium citrate as co-coordinating agents through a hydrothermal reaction. In brief, $1 \mathrm{~mL}$ of oleic amine (OA) was dissolved in $10 \mathrm{~mL}$ of ethylene glycol (EG, J. T. Baker) to form a clear solution; next, $10 \mathrm{mmol}$ of iron (III) chloride hexahydrate $\left(\mathrm{FeCl}_{3} \cdot 6 \mathrm{H}_{2} \mathrm{O}\right.$, Acros) and $20 \mathrm{mmol}$ of sodium acetate anhydrous (NaAc, Aldrich) were added. Then, the mixture was mixed at $60{ }^{\circ} \mathrm{C}$ for $24 \mathrm{~h}$ to generate a homogeneous solution. The mixture was placed in a Teflon-lined stainless steel autoclave for hydrothermal reactions at $220{ }^{\circ} \mathrm{C}$ for $8 \mathrm{~h}$. After the reaction, the autoclave was cooled down to room temperature for another $3 \mathrm{~h}$. The magnetic particles were collected by centrifugation at 7,000 rpm for 12 min and washed with excess ethanol 3 times afterwards. Before characterization and application, the particles were dried under vacuum overnight at room temperature. To investigate the capping ability of lactoferrin (Lf), OA-coated MIONs were dissolved in DI water $(0.5 \mathrm{mg} / \mathrm{mL})$ in advance. To provide linkers, the MION suspension was vigorously stirred at $4{ }^{\circ} \mathrm{C}$ for $3 \mathrm{~h}$ with $40 \mu \mathrm{L}$ of 1-ethyl-3-(3-dimethylaminopropyl) carbodiimide (EDC, Sigma) and $40 \mu \mathrm{L}$ of N-hydroxysuccinimide (NHS, Sigma-Aldrich) (0.1\%). The particles were washed using DI water by centrifugation at 8,500 rpm 3 times and were then redispersed in the DI water. Lf at various concentrations and dissolved in DI water was added to the solution, which was stirred continuously for another $24 \mathrm{~h}$ at 4 ${ }^{\circ} \mathrm{C}$ to allow for protein conjugation to MIONs. The resulting product was rinsed with DI water for later use.

ZnS-Capped CdSe quantum dots (QDs, Oceananotech) were loaded in OA-coated MIONs for tracking and imaging purposes. Loading QDs in OA-coated MIONs was achieved by dispersing OA-coated MIONs in a solvent mixture containing 5\% $(\mathrm{v} / \mathrm{v})$ chloroform and 95\% (v/v) n-butanol, and then by adding a controlled amount of QDs to the mixture. 
After vortexing and stirring for $2 \mathrm{~h}$, the particles were collected by centrifugation at $6,000 \mathrm{rpm}$ for $10 \mathrm{~min}$ and re-dispersed into ethanol. Then, the product was washed with excess ethanol by three times and collected by centrifugation at $6,000 \mathrm{rpm}$ for $10 \mathrm{~min}$ afterward. The resulting QD-loaded MIONs were dispersed in D.I. water for further modification.

\section{Characterization}

The morphologies of MIONs and Lf-MIONs were characterized using a field emission scanning microscope (FE-SEM, JEOL-6700, Japan). For the microscope observation, the particles were dried on a silicon wafer and coated with platinum through sputtering for 90 seconds. An X-ray diffractometer (XRD Bruker AXS, XRD-D8) was used to estimate the phase of the particles by scanning over $2 \theta$ from $10^{\circ}$ to $70^{\circ}$ under $\mathrm{Cu} \mathrm{Ka}$ radiation. Furthermore, to investigate the magnetic properties and hysteretic behavior of MIONs and Lf-MIONs, a superconducting quantum interference device (SQUID, MPMS-XL) was used from $-10,000 \mathrm{G}$ to $+10,000 \mathrm{G}$ at $298 \mathrm{~K}$. A high-frequency magnetic field (MF) was used to induce the heating of MIONs. The suspension of MIONs or Lf-MIONs was placed into tubes and subjected to MF at various strengths $(4,8$, and $16 \mathrm{kA} / \mathrm{m})$. After the heating, the temperature was measured using a thermometer.

\section{Perfluorohexane (PFH) and fluorescent dye loading and release}

Perfluorohexane (PFH) and a fluorescent dye (rhodamine dye) were used to investigate the encapsulating and releasing behaviors of MIONs and Lf-MIONs at various conditions. In brief, the PFH and dye were mixed via sonication of the particles at room temperature and sealed in bottle. Due to the hydrophobic-hydrophobic affinity, the PFH and dye were absorbed on the pores of particles. Then, the free PFH and dye were removed by centrifugation at 5,000 rpm for $10 \mathrm{~min}$ and washed with excess DI water three times. To evaluate the loading content of PFH, the particles encapsulating PFH were kept at a different temperature to evaporate the loaded $\mathrm{PFH}$, and then, the particles were re-weighed. The amount of PFH was calculated using the weight loss of $\mathrm{PFH}$ during the evaporation. Rhodamine dye was used to investigate the releasing property at various conditions, where the dye, which functioned as a model drug in the release experiment, could be observed using an inverted fluorescence microscope (ECLIPSE Ti, NIKON, Japan), and the fluorescence intensity was quantified using a fluorescence spectrophotometer (F-7000, Hitachi, Japan). To determine the loading capacity of dye, the dye was extracted from dye-loaded particles by methanol. The concentration of dye was quantified using a fluorescence spectrophotometer (F-7000, Hitachi, Japan). Then, dye loading capacity can be determined after calculation.

\section{Drug loading and release}

To load Paclitaxel (PTX, Scinopharm, Taiwan), 2 $\mathrm{mg}$ of PTX was dissolved in $2 \mathrm{~mL}$ of DMSO, and different carriers were soaked in a solution, followed by stirring for $24 \mathrm{~h}$. Then, the drug-encapsulated carrier was dried under vacuum to evaporate the DMSO for another $24 \mathrm{~h}$. Then, phosphate-buffered saline (PBS) was used to wash the carriers 3 times to remove un-loaded PTX.[34] To prepare the PTX/MIONs-PFH, $\mathrm{PFH}$ was mixed with the particles at room temperature for $2 \mathrm{~h}$, and then, the particles were washed to remove the free PFH by centrifugation at 5,000 rpm for 10 min with excess DI water. Furthermore, Lf was used to cap PTX/MIONs-PFH through EDC reactions for $3 \mathrm{~h}$. The resulting particles were washed using DI water through centrifugation at 8,500 rpm 3 times and then re-dispersed in DI water and stored at $4{ }^{\circ} \mathrm{C}$. To determine the loading capacity of PTX, PTX was extracted from $10 \mathrm{mg}$ of drug-loaded particles by $10 \mathrm{~mL}$ acetonitrile. High-performance liquid chromatography (HPLC, Agilent Technologies 1200 series) with a $150 \mathrm{~mm}$ ZORBAX Eclipse XDB-C18 (5 $\mu \mathrm{m})$ column was used to quantify the PTX at a wavelength of 204 $\mathrm{nm}$; the mobile phase was $40 \%$ water and $60 \%$ acetonitrile. Then, the loading capacity was calculated while identifying the concentration of PTX. The encapsulation efficiency (EE \%) was determined by the following equation: $\mathrm{EE} \%=(\mathrm{A}-\mathrm{B}) / \mathrm{A} \times 100$, where $\mathrm{A}$ is the total amount of PTX, and B is the amount of PTX remaining in the supernatant. Furthermore, GC was applied to quantity the PFH content after PFH was extracted by chloroform from particles.

The in vitro drug release of PTX was examined in $0.1 \%$ Tween-80 (v/v) in $20 \mathrm{~mL}$ of DI water. Then, 3 $\mathrm{mL}$ of released solution with dispersed PTX-loaded carrier was collected and separated by centrifugation at $6000 \mathrm{rpm}$ for $10 \mathrm{~min}$. The clear supernatant containing released PTX was characterized using HPLC, and the measurement was performed in triplicate.

Similarly, high-frequency magnetic field (MF)-simulated PTX release was identified with different MF treatment conditions. MF with a frequency of $50 \mathrm{kHz}$ can be used to activate/heat the MIONs and Lf-MIONs owing to the magnetically induced energy. A suspension of drug-loaded MIONs and Lf-MIONs was placed into tubes and treated with MF of different magnitudes $(0,4$, and $8 \mathrm{kA} / \mathrm{m})$. After the treatment, a thermometer was used to measure the temperature, and the drug release profile was determined by HPLC. 


\section{Cytotoxicity and in vitro cell uptake}

RG2 (a brain cancer cell line) was maintained in Eagle's minimum essential medium containing $10 \%$ fetal bovine serum and $1 \%$ penicillin/streptomycin in a $5 \% \mathrm{CO}_{2}$-enriched atmosphere at $37{ }^{\circ} \mathrm{C}$. After the cells were cultured for $24 \mathrm{~h}$, various carriers were added to the cancer cells for different times. The cell viability after different treatments was investigated by a counting method based on trypan blue exclusion. After removal with trypsin, cells were collected and scored using optical microscopy. The surviving cells (unstained by trypan blue) were counted up to 5 times, and three individual experiments were carried out for various treatment groups. To estimate the cellular uptake of carriers using confocal laser scanning microscopy, hydrophobic fluorescent quantum dots (Oceantech, USA) were loaded into the pores of carriers through hydrophobic interactions. Then, RG2 cells were incubated with QD-loaded MIONs and Lf-MIONs for different times. The cells were observed with a confocal microscope (CLSM, Zeiss LSM 700 Confocal, Germany). Additionally, the cells were harvested by trypsin-EDTA and resuspended in medium for flow cytometry analysis $(10,000$ cumulative events, analyzed by CELLQUEST ${ }^{\circledR}$ software).

Three-dimensional tumor spheroid models were prepared by using a liquid overlay method by coating $1 \mathrm{wt} \%$ agarose on cell dishes in phosphate-buffered saline (PBS). RG2 cells were incubated at $2 \times 10^{5}$ cells per well in $4 \mathrm{~mL}$ of cell culture medium with $10 \%$ fetal bovine serum and $1 \%$ penicillin/streptomycin in a $5 \% \mathrm{CO}_{2}$-enriched atmosphere at $37^{\circ} \mathrm{C}$. After $48 \mathrm{~h}$, tumor spheroids that were approximately $200 \mu \mathrm{m}$ in diameter were formed. To observe the spheroids, the spheroids were fixed with $1 \%$ formaldehyde and permeabilized with $0.1 \%$ Triton X-100 for $30 \mathrm{~min}$. Rhodamine phalloidin and DAPI were applied to stain the F-actin and nuclei of cells, and the spheroids were imaged using a confocal laser scanning microscope (CLSM, Zeiss LSM 780 Confocal, Germany). The CLSM software (ZEN lite) was used to analyze and quantify the fluorescence intensity from various signals. Furthermore, the effects of MF on the tumor spheroids were also evaluated by CLSM. After the tumor spheroids formed, PFH/Lf-MIONs were incubated with spheroids for another day. Subsequently, an MF with a strength of $4 \mathrm{kA} / \mathrm{m}$ was applied to the cells for 30 seconds. Then, the cells were stained and observed using identical approaches for CLSM.

\section{In vitro magneto-thermo-chemo-therapy}

RG2 cells and tumor spheroids were incubated with MIONs/PTX, Lf-MIONs/PTX, and Lf-MIONs/PTX-PFH for $24 \mathrm{~h}$, and then, the cells were washed to remove the free particles. The loading ca- pacity of PTX in Lf-MIONs/PTX and Lf-MIONs/PTX-PFH was kept at $0.5 \mathrm{mg} \mathrm{PTX} / \mathrm{mg}$. The cell viability was investigated by a counting method. Trypsin was used to remove the cells from dish, and trypan blue was applied to distinguish the live/dead cells. Then, the cells were counted and scored using an optical microscope. The individual experiments were carried out 4 times for each treatment group. Similarly, the effects of high-frequency magnetic field (MF)-simulated PTX release was also evaluated. An MF with a strength of $8 \mathrm{kA} / \mathrm{m}$ was used to treat the cells and tumor spheroids for $5 \mathrm{~min}$. After the treatment, the cells were incubated for another $24 \mathrm{~h}$. Again, the cell viability was estimated using an identical method.

\section{In vivo anti-tumor efficacy}

All animals were obtained from BioLASCO Taiwan Co. (Taiwan) and handled in accordance with the guidelines for Animal Care and Use Committee of the Institute of Life Science of National Tsing Hua University. To induce solid tumors, RG2 cells $\left(1 \times 10^{5}\right.$ in $100 \mu \mathrm{L}$ PBS) were injected into female nude mice (CAnN.Cg-Foxn) that were 6 weeks old. When the tumor volume reached $50 \mathrm{~mm}^{3}, 100 \mu \mathrm{L}$ of saline solution containing $2 \mathrm{wt} \%$ carriers was i.v. injected into the mice through the tail vein. To evaluate the effect of chemotherapy, post-treated tumor sizes were measured at different time points using a digital caliper and photography. Parallel studies of magneto-thermo-chemo-therapy used mice that had received $1 \mathrm{MF}$ treatment $(4 \mathrm{kA} / \mathrm{m})$ for $10 \mathrm{~min}$ at $24 \mathrm{~h}$ post-injection. After 16 days, the tumors were collected and weighed after fixing with $10 \%$ formalin. All measurements were performed in triplicate.

\section{Results}

\section{Synthesis and characterization of Lf-MIONs}

Mesoporous iron oxide nanoparticles (MIONs) are fabricated via a facile ligand-assisted synthesis method by the introduction of oleic amine (OA) and sodium citrate as co-coordinating agents through a hydrothermal reaction. In Figure $\mathbf{2 a}$, the synthesized MIONs with a diameter of approximately $160 \mathrm{~nm}$ can be uniformly dispersed in aqueous solution. The porous structures can also be clearly observed for each particle. The higher magnification in Figure $\mathbf{2} b$ shows that a MION is composed of several iron oxide domains that are approximately $15 \mathrm{~nm}$ in diameter, and the MION pores are constructed between these iron oxide domains. The possible MION formation mechanism can be explained as two steps during the hydrothermal synthesis. First, $\mathrm{Fe}^{3+}$ ions are partly reduced to $\mathrm{Fe}^{2+}$ by ethylene glycol, and then, $\mathrm{Fe}_{3} \mathrm{O}_{4}$ 
primary nanoparticles were formed via dehydration of these ions. Second, the formation of MIONs seems plausible through the oriented attachment of these primary particles; this orientation can minimize their surface energy and magnetic dipole alignment. After the OA removal step in the synthesis process, the particle size increases, and the pores between each iron oxide domain become indistinct, as demonstrated in Supplementary Material: Figure S1. The OA-ligand complex on the surface of the pores restricted particle growth and led to a pore hydrophobicity, which promoted the effective encapsulation of hydrophobic paclitaxel (PTX) and perfluorohexane (PFH) molecules in the carrier. Furthermore, these hydrophobic pores of MIONs can be further capped by a thermal-sensitive bio-gate (a biocompatible protein, lactoferrin (Lf)) through absorption and partial covalent bonds [34-36]. Concerning Lf, we found a lower concentration Lf (e.g., $1 \mathrm{wt} \% \mathrm{Lf}$ ) favored the deposition of a thin layer to cap MIONs (Figure 2c, 2d), and a higher concentration Lf (e.g. $5 \mathrm{wt} \%$ Lf) favored filling the whole pore (Figure $\mathbf{2 e}, \mathbf{2 f}$ ). This result implies a high affinity between pores and Lf due to hydrogen bond interactions. Furthermore, after the chemical linking, the Lf coating persists for more than one month without desorption, indicating the good stability of Lf on the pores. The closer observation in Figure $2 \mathbf{d}$ shows that after the application of a low-concentration Lf coating, the ultrathin layer of Lf on MION is approximately a few nanometers in thickness, and no observable crevices or cracks were microscopically detectable on the surface of the Lf-MIONs, suggesting a compatible interface between these two components. Once the concentration of Lf increased, the pores filled, as indicated by the observed smooth surface (Figure 2e and 2f). Furthermore, the results of zeta potential analysis and thermogravimetric analysis (TGA) further proved that Lf conjugated to the MIONs. The charge of MIONs was changed from positive $(+10.8 \mathrm{mV})$ to negative $(-12.4$ $\mathrm{mV}$ ) after the Lf conjugation to MIONs, as measured by the zeta potential. Moreover, TGA analysis indicated that the weight ratio of Lf in Lf-MIONs is approximately $4.6 \%$ (Figure $2 \mathrm{~g}$ ). Furthermore, to confirm the chemical bonds, X-ray photoelectron spectroscopy (XPS) was applied. As shown in Supplementary Material: Figure S2, the binding energy of $\mathrm{O}$ 1s is detected from 527.0 to $537.0 \mathrm{eV}$, and the peak of iron oxide was at $529.3 \mathrm{eV}$, which is consistent with literature report. A peak at $530.4 \mathrm{eV}$ for the Lf indicates its carboxylic acid group. When Lf and OA-MIONs were incorporated, a shifting to higher energy region $(531.4 \mathrm{eV})$ was observed, implying that the carboxylic acid was chemically bonded or immobilized onto the particle surface. The higher binding energy is believed to be a result of EDC reaction between primary amine on the surface of the MIONs and carboxylic acid group of Lf, forming a covalent bonding, implying an excellent chemical affinity between both participating phases and a mechanically strong solid network is achieved. Beside, after conjugating, a peak at $288.1 \mathrm{eV}$ of $\mathrm{C} 1 \mathrm{~s}$ for amide bonds $(\mathrm{O}=\mathrm{C}-\mathrm{N})$ increased, which further confirms the formation of bonding between OA-MIONs and Lf. For long-term preservation, the Lf-MIONs were freeze-dried at $-80{ }^{\circ} \mathrm{C}$. The freeze-dried particles can be resuspended in water and showed the same dispersion and weight loss as before. This finding suggests that the drug carriers remained unchanged during freeze-drying, which can preserve Lf-MIONs and prevent drug release. We note that the Lf-MIONs can be used as MRI contrast agents, which is outside the scope of this study.

The choice of capping protein for the drug delivery platform is also motivated by consideration of the in vivo long-term circulation. Most carriers are known to absorb serum proteins during in vivo circulation, which significantly induces the uptake of nanocarriers by macrophages. As a result, carriers are removed by clearance organs, and only a few carriers reached the targeted tumor. Recently, surface modification, such as polyethyleneglycol (PEG) coating, of these nanocarriers has become an attractive technique to prevent this situation. However, many natural particles, such as lipoproteins coated by apolipoproteins, can also eliminate the uptake of macrophages. Therefore, a protein shell on the nanoparticle can theoretically reduce unintended serum protein coating. For this approach, the Lf-coated MION (termed Lf-MION) is a first attempt to use protein-capped porous nanoparticles to improve functionality. Moreover, compared to other mesoporous particles, the Lf-MIONs carrying large amounts of hydrophobic drug and perfluorohexane (PFH) in their pores display little drug and gas release until the particles are actuated by a high-frequency magnetic field (MF). Because MF-responsive MIONs can generate intense heat locally, both PFH gasification and magneto-thermo-chemo-therapy guide local particles with enhanced permeability to deep tumor cells and enable the release and spread of a large dose of PTX to kill tumor cells.

X-ray diffraction (XRD) patterns revealed that the crystalline phase of $\mathrm{MIONs}$ is $\mathrm{Fe}_{3} \mathrm{O}_{4}$ (magnetite), as shown in Figure $\mathbf{2 h}$. Six main diffraction peaks at $2 \theta=30^{\circ}, 36^{\circ}, 43^{\circ}, 54^{\circ}, 57^{\circ}$, and $63^{\circ}$ that can be considered the characteristics peaks of $\mathrm{Fe}_{3} \mathrm{O}_{4}$ crystal plane can be detected (according to $\mathrm{Fe}_{3} \mathrm{O}_{4}$ (JCPDS [85-1436])). After the MIONs were coated with Lf, the relative diffraction intensity of the $\mathrm{Fe}_{3} \mathrm{O}_{4}$ peaks be- 
came weaker because the presence of the amorphous protein lowered the concentration of iron oxide nanoparticles. A superconducting quantum interference device (SQUID) at $298 \mathrm{~K}$ with the magnetic field sweeping from $-10,000$ to $+10,000 \mathrm{G}$ was used to evaluate the magnetic property of MIONs, oleic amine-coated MIONs (OA-MIONs), and lactoferrin coated-MIONs (Lf-MIONs). In Figure 2i, the correlations between the magnetizations of the MIONs, OA-MIONs and Lf-MIONs and the magnetic field have a similar shape and negligible hysteresis. Again, the presence of oleic amine and lactoferrin dilutes the MIONs, leading to a lower saturation magnetization (Ms) of the Lf-MIONs compared with that of the pure MIONs. A magnet that was near the solution could completely attract these particles, indicating that the Lf-MIONs possess excellent magnetic response (inset picture of Figure 2i). Furthermore, the magnetic property of these Lf-MIONs after storage at $4{ }^{\circ} \mathrm{C}$ for 3 weeks was investigated, as shown in Supplementary Material: Figure S3. The result reveals no obvious change in the magnetic characteristics.
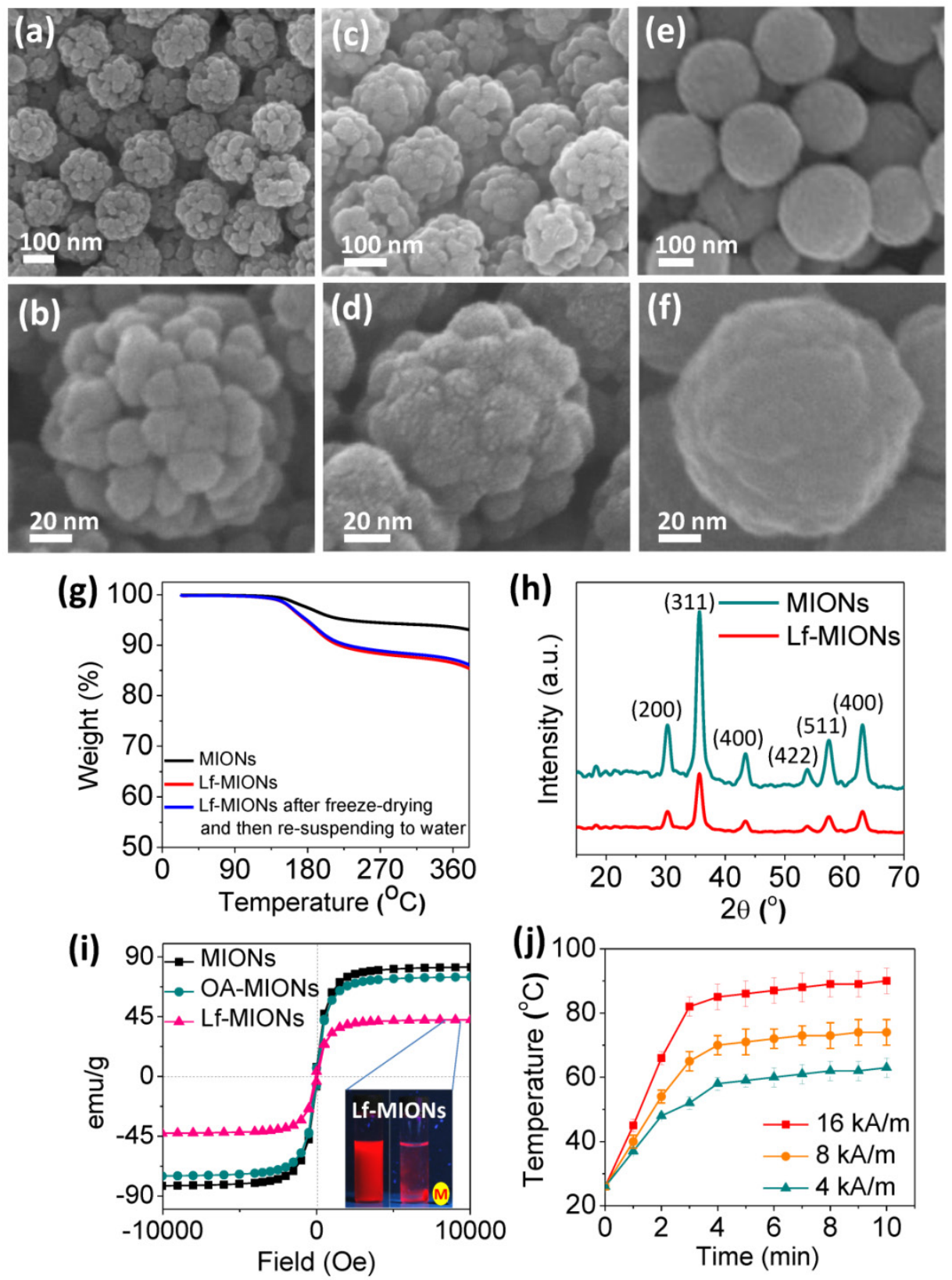

Figure 2. SEM images of Lf-MIONs prepared using various concentrations of lactoferrin for coating with different magnifications: (a, b) 0 \%, (c, d) 1 \%, and (e, f) $2 \%$. (g) Thermogravimetric analysis (TGA) analysis of MIONs and Lf-MIONs, and the weight loss of Lf-MIONs after freeze-drying and resuspension in water. The dried Lf-MIONs can be re-dispersed in water solution and displayed similar weight loss as before, suggesting that the Lf-MIONs remained intact during the drying preservation and precluding any possibility of Lf desorption. (h) X-ray diffraction patterns of as-synthesized MIONs and Lf-capped MIONs (Lf-MIONs). (i) Field-dependent magnetization curve of MIONs, oleic amine-coated MIONs (OA-MIONs) and Lf-MIONs. (j) Heat generation kinetics of a PBS suspension of Lf-MIONs for 4, 8 and 16 kA/m high-frequency magnetic fields (MFs). 
When Lf-MION suspensions (0.1 mg/ $\mathrm{mL}$ in PBS) were treated with a high-frequency magnetic field (MF) with a frequency of $50 \mathrm{kHz}$, an obvious increase in temperature was measured (Figure $\mathbf{2} \mathbf{j}$ ) for various MF intensities ranging from 4 to $16 \mathrm{kA} / \mathrm{m}$. The mechanism by which the MF induces heat from magnetic particles is through magnetic energy dissipation (Brown and Néel relaxations), which is detrimental to the crystal size and the materials [37,38]. The temperature increase in Lf-MIONs can be regulated by the MF strength and treatment time. When the strength of MF was $16 \mathrm{kA} / \mathrm{m}$, the temperature of the solution rose to $80{ }^{\circ} \mathrm{C}$ within $3 \mathrm{~min}$, indicating excellent sensitivity of the MIONs. Even for a low-strength $\mathrm{MF}(4 \mathrm{kA} / \mathrm{m})$, the temperature increased to $50{ }^{\circ} \mathrm{C}$ rapidly. Such highly sensitive behavior of heat generation is convenient for use in tumor thermo-therapy, especially for deep tumors. Because the MF strength can be gradually decreased by adjusting the induction coil, the high sensitivity of heat generation induced by a weak high-frequency MF is extremely important for clinical uses.

\section{Encapsulation and controlled release of PFH and PTX}

To evaluate the encapsulation of perfluorohexane $(\mathrm{PFH})$ and the temperature response, PFH encapsulated in Lf-MIONs was vaporized and observed using confocal laser scanning microscopy (CLSM) (both $\mathrm{PFH}$ and red fluorescent dye were embedded in Lf-MIONs). The PFH in the pores of Lf-MIONs is stabilized by the hydrophobic/hydrophobic interactions between PFH and the large surface areas of oleic amine-modified pores. As expected, the bubbles were visualized when the heat treatment was applied to $\mathrm{PFH} / \mathrm{Lf}-\mathrm{MIONs}$ at $75^{\circ} \mathrm{C}$ for $1 \mathrm{~s}$ (Figure 3a). This result implied that PFH has been successfully encapsulated into the MIONs and exhibited heat responsiveness. Additionally, the red fluorescent dye (rhodamine dye as a model drug) embedded in Lf-MIONs was released rapidly upon PFH gasification and then diffused through the solution. To evaluate the influence of temperature on the PFH/Lf-MIONs, the carriers were maintained at 37,45 and $75{ }^{\circ} \mathrm{C}$ to evaporate the encapsulated $\mathrm{PFH}$. The carriers were re-weighed at regular intervals, as shown in Figure $3 \mathrm{~b}$. At $37^{\circ} \mathrm{C}$, the amount of PFH slightly decreased at $60 \mathrm{~min}$. In contrast, at $45{ }^{\circ} \mathrm{C}$, the encapsulated $\mathrm{PFH}$ was fully released after $60 \mathrm{~min}$, and at $75^{\circ} \mathrm{C}$, all the $\mathrm{PFH}$ was lost in $30 \mathrm{~min}$. The results suggested that the higher temperature provided stronger energy for inducing PFH vaporization. The dependence of in vitro fluorescent dye release on the temperature increase in Lf-MIONs was also considered, as shown in Figure 3c. Compared to the situation at $37^{\circ} \mathrm{C}$, when the temperature was kept at $75{ }^{\circ} \mathrm{C}$, the dye release rate increased significantly within $5 \mathrm{~min}$, indicating the rapid thermal response of Lf-MIONs. Subsequently, the sustained fast release was maintained for $40 \mathrm{~min}$. After $40 \mathrm{~min}$, another sustained release behavior was measured, and more than $74 \%$ of the dye was released. At $45^{\circ} \mathrm{C}$, the release rate was slower than that at $75{ }^{\circ} \mathrm{C}$, but a similar release pattern was observed. These results are significantly agreement with Figure $\mathbf{3 b}$ but also show that the dye release rate is slower than the predicted value, which suggests that dye molecules cannot be completely released. The uncompleted release is probably due to the strong interactions between dyes and particle surfaces. Because the mesoporous iron oxide nanoparticle is not a thermosensitive material, it is believed that the PFH vaporization and Lf deformation contributed to the dye release. Thus, we can conclude that the release behaviors of Lf-MION are highly dependent on the influence of temperature. (a)

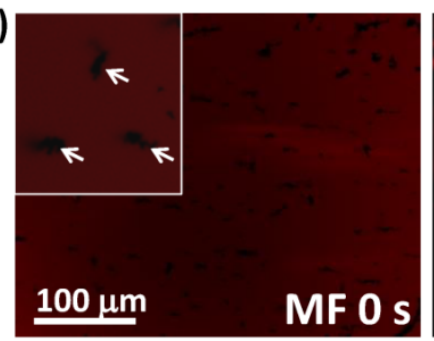

(c)

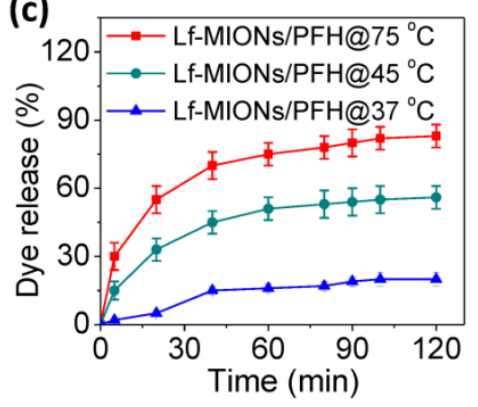

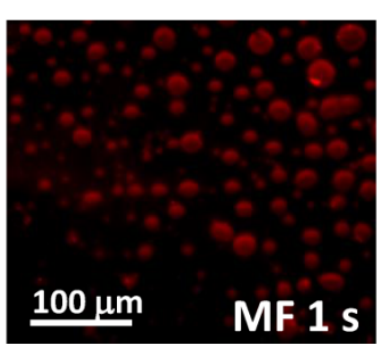

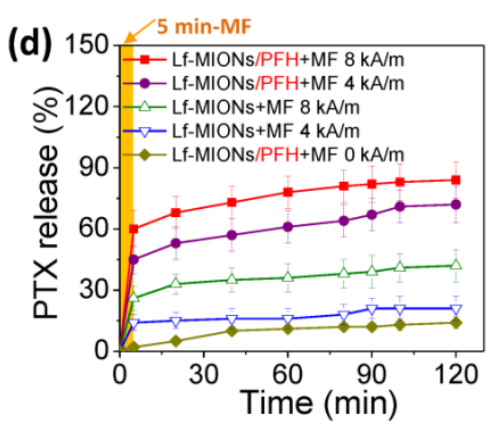

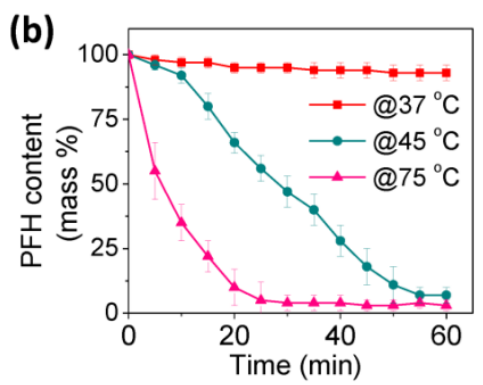

(e)

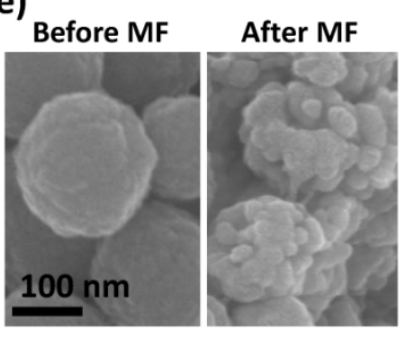

Figure 3. (a) Confocal laser scanning microscopy (CLSM) images of Lf-MIONs encapsulating perfluorohexane (PFH) before and after heating at 75 ${ }^{\circ} \mathrm{C}$ for 1 second. White arrows in the inset pictures point to the Lf-MIONs. (b) The PFH content patterns monitored by thermogravimetric traces over time at $37{ }^{\circ} \mathrm{C}, 45{ }^{\circ} \mathrm{C}$, and 75 ${ }^{\circ} \mathrm{C}$. (c) Cumulative dye (rhodamine dye as a model drug) release from Lf-MIONs at various temperatures. (d) Cumulative PTX release from Lf-MIONs after $5 \mathrm{~min}$ of a high-frequency magnetic field (MF) treatment at 0,8 , and 16 $\mathrm{kA} / \mathrm{m}$. (e) SEM image of Lf-MIONs after MF treatment. 
A hydrophobic anti-cancer drug, paclitaxel (PTX), was encapsulated by MIONs, and the loading capacity of PTX in MION reached $0.92 \mathrm{mmol} \mathrm{g}{ }^{-1}$. When compared to MIONs without an oleic amine modification, these MIONs showed lower PTX loading capacity $\left(0.53 \mathrm{mmol} \mathrm{g}^{-1}\right)$. The large payload of PTX is not solely caused by the surface area alone; it also reflects the affinity between PTX and hydrophobic pore surfaces. Furthermore, it is well known that a nanoporous structure is a potent absorbent of oil-soluble drugs [39]. Therefore, the MIONs with the OA modification exhibited higher loading capacity than those without OA modification. To confirm the pore structures of OA-MIONs before and after PTX loading, BET and TEM were applied as shown in Supplementary Material: Table S1, Figure S4, and Figure S5. The characteristics pore filling step disappears in the $\mathrm{N}_{2}$ adsorption isotherm after PTX is stored, and the pore volume decreases by about $80 \%$, which indicates that most of the pores have been filled. From the TEM results in Supplementary Material: Figure $\mathbf{S 5 a}$ and $\mathbf{S 5 b}$, the porous structure can be obtained for OA-MIONs. However, after loading PTX to OA-MIONs, most of the pores were filled as shown in Supplementary Material: Figure S5c and S5d. After PTX loading, the MION-PTX system was loaded PFH and then capped by Lf bio-gates. Figure $\mathbf{3 d}$ shows profiles of the PTX release from Lf-MIONs under the influence of a high-frequency magnetic field (MF) at strengths of 0,4 and $8 \mathrm{kA} / \mathrm{m}$. The results demonstrated that the PTX release rate for MF treatment is much faster that that without MF treatment. The PTX release from Lf-MIONs is less than $15 \%$ in $120 \mathrm{~min}$. For Lf-MIONs subjected to MF for $5 \mathrm{~min}$ at strengths of 4 and $8 \mathrm{kA} / \mathrm{m}$, the PTX release can be controlled: the stronger the MF treatment is, the more drugs are released. When $5 \mathrm{~min}$ of MF was used, the PTX release of Lf-MIONs followed a burst-like release pattern, and then the release rate decreased after MF removal. As demonstrated in Figure $\mathbf{2} \mathbf{j}$, MF can induce intense heat through magnetic energy dissipation $[37,38]$. Such strong energy leads to the release of PTX from Lf-MIONs due to a combination of the PFH gasification and Lf conformation changes. Here, Lf is used as thermosensitive polymer. Because the temperature is increased to approximately $42{ }^{\circ} \mathrm{C}$, most hydrogen bonds between peptides disappear, which loosens the intramolecular interactions between protein structures. The behavior was confirmed by SEM imaging (Figure 3e), which showed that the surface of Lf-MIONs became rough after MF treatment; this changed indicated that the Lf structures became deformed. Therefore, for the MF treatment (4 and 8 $\mathrm{kA} / \mathrm{m}$ ), the unrecoverable deformation of bio-gates still showed a sustained release after removal of the
MF treatment. Additionally, to understand the PTX stability of Lf-MIONs/PTX-PFH, the cumulative PTX release without MF treatment was also examined as shown in Supplementary Material: Figure S6. The hydrophobic PTX exhibits little driving force for dissolution in the water, so it suffers from little release except for the step release in the beginning which may be attributed to the desorption of the residual PTX on particles surfaces. Both release profiles of Lf-MIONs/PTX-PFH and Lf-MIONs/PTX are less than $15 \%$ in $120 \mathrm{~min}$, suggesting the good stability of PTX in MIONs.

\section{Cell viability and uptake}

To investigate the cell viability of MION and Lf-MION, carriers with various concentrations were incubated with RG2 cells (a brain cancer cell line) for $24 \mathrm{~h}$. At concentrations up to $5 \mathrm{mg} / \mathrm{mL}$ of MION, the results demonstrated that the drug-free MION (cell viability of approximately $82 \%$ ) and Lf-MION (cell viability of approximately $87 \%$ ) caused low toxicity to cells (see Supplementary Material: Figure S7). Next, for an evaluation of the RG2 cell uptake efficacy of carriers, quantum dots (QD) with emission fluorescence at $600 \mathrm{~nm}$ were loaded into the hydrophobic pores of MION and Lf-MION to enable tracking in the cells. Lf is a common targeting ligand for brain tumors because Lf receptors are overexpressed on RG2 cells [35]. After $30 \mathrm{~min}$ of incubation with Lf-MION, Lf-MIONs that were highly labeled with QD (red dots) were observed using confocal laser scanning microscopy imaging, and most of Lf-MIONs were found in the cytoplasm, indicating the high targeting and internalization ability (Figure $4 a$ ). Figure $4 b$ and $4 \mathrm{c}$ shows the results for 1 and $2 \mathrm{~h}$ of incubation with Lf-MION, further indicating that the cytoplasm contains these particles and the enhancement of the fluorescence signal in the cells. For comparison, MIONs without Lf capping were also incubated with RG2 cells in the control group. As shown in Figure 4d to $4 \mathbf{f}$, the accumulation of MIONs is relatively low for similar incubation times, especially for $30 \mathrm{~min}$, suggesting a weak interaction between the particles and the cell surfaces. Furthermore, flow cytometry was used to quantify the cell uptake of MION and Lf-MION in an RG2 cell line (Figure $\mathbf{4 g}$ ). The fluorescence intensity from the Lf-MIONs is approximately 2 times stronger than that from the MIONs without Lf after $30 \mathrm{~min}$ of incubation, indicating that the Lf receptors increased the internalization. For an increase in the incubated time to $2 \mathrm{~h}$, the fluorescence intensity significantly increased because of the cell uptake of Lf-MIONs. Therefore, the Lf surfaces on MIONs can enhance cell uptake. A control experiment in which RG2 cells were incubated with QD-free Lf-MIONs confirmed that the 
red fluorescence is from the QDs in Supplementary Material: Figure S8. Another cell line with low Lf receptor overexpressing, MRC-5 (a human lung fibroblast, normal cell) cell, is also incubated with Lf-MIONs, exhibiting no obvious cell uptake of Lf-MIONs (Supplementary Material: Figure S9).

\section{Evaluation of Lf-MIONs in in vivo-like multi- cellular tumor spheroids}

The in vivo-like multicellular tumor spheroids was applied to understand the intratumoral behaviors of nanoparticle penetration, cellular uptake and drug molecular diffusion from delivery systems in real time [24]. With results similar to those for an in vivo solid tumor, many studies have demonstrated that such tumor spheroids display heterogeneous cell morphologies, which differ depending on the relative positions of the spheroids: the cells around the periphery are mainly proliferating, and the cells in the center are usually necrotic and apoptotic [40-43]. The peripheral edge of a tumor spheroid is composed of a high density of compact cancer cells, which poten-
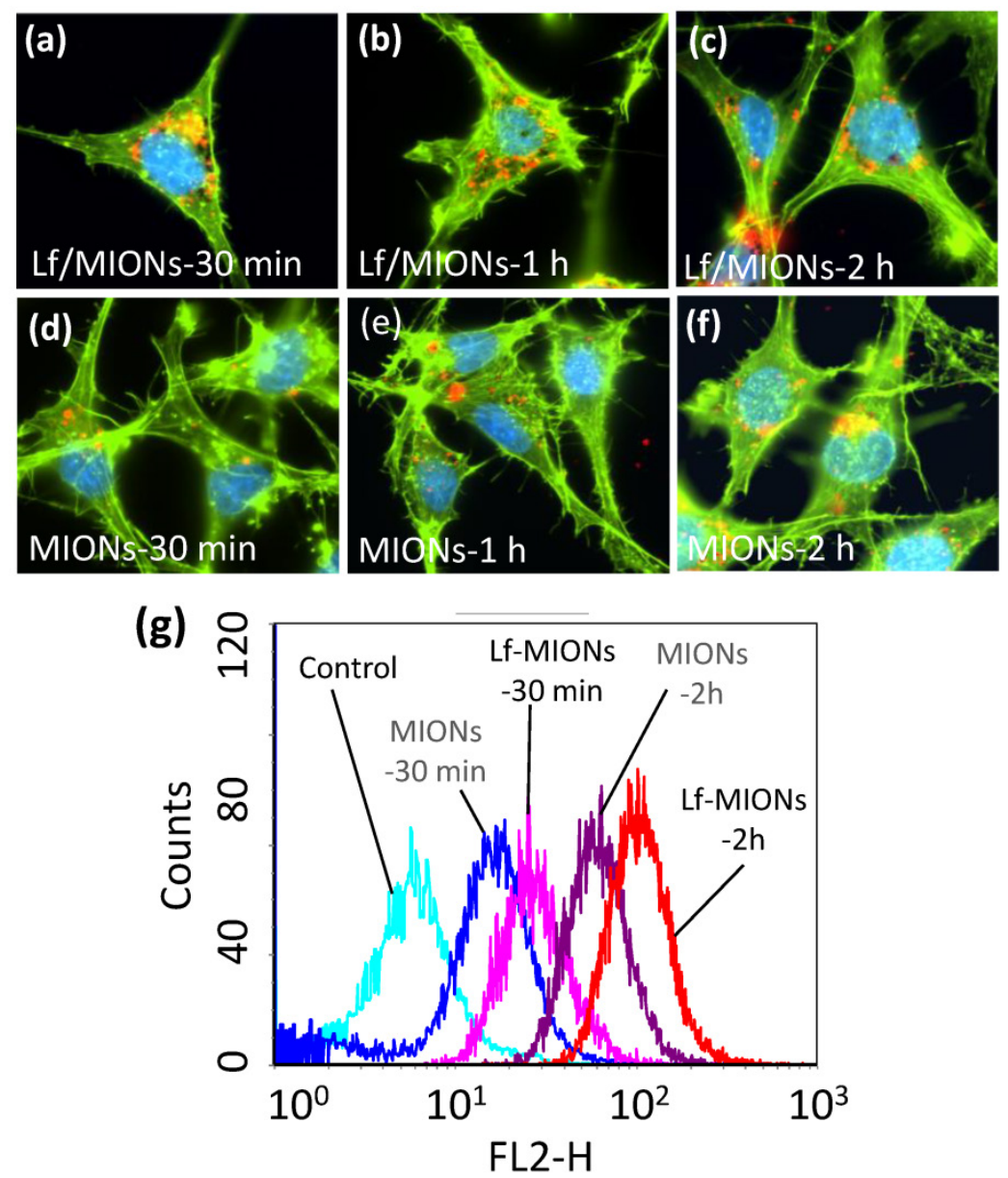

Figure 4. (a-f) Cellular uptake of quantum dot (QD)-loaded (a-c) Lf-MIONs and (d-f) MIONs in RG2 cells after $30 \mathrm{~min}, 1 \mathrm{~h}$, and $2 \mathrm{~h}$ of incubation: the QD red fluorescence increases over time from many cell regions, including the cytoplasm. (g) Flow cytometry analysis of QD-loaded Lf-MIONs after various incubation times in RG2 cells. tially block the penetration of most molecules and particles to the center. As shown in Figure 5a, a 3D tumor spheroid is approximately $200 \mu \mathrm{m}$ in diameter and has a clear boundary between the outer and inner regions of the spheroid when observed under a confocal laser scanning microscope (CLSM). Moreover, the peripheral edge of the tumor spheroid exhibited strong green fluorescence from the cell skeleton, suggesting that the peripheral cells formed a dense wall similar to that of a real tumor. In Figure 5a, without the assistance of Lf-targeting ligands, only a few MIONs attached to the tumor spheroid, and no particles were observed in the center after $2 \mathrm{~h}$ of incubation. Figure $\mathbf{5 b}$ represents the mean fluorescence intensity profiles from the cell skeleton (green line) and QD-labeled MIONs (red line) calculated from 10 tumor spheroids. The normalized radius values of 0 and 1 on the $x$-axis mean the position of the center and shell, respectively, of the tumor spheroid. Consistent with the results of the CLSM image, the fluorescence intensity of MIONs is weak, suggesting only a few particles in the spheroids. In contrast, when Lf-MIONs were applied to the tumor spheroid, the tumor cells took up a large amount of particles, but most of them still resided in the outer region of tumor spheroids (Figure $\mathbf{5 b}$ and $\mathbf{5 e}$ ). The cell uptake behaviors are similar for those of a two-dimensional cell culture. However, after the tumor spheroid was treated with Lf-MIONs/PFH, the particles with bright red fluorescence penetrated the inner regions from the surface to the middle (Figure 5c). Moreover, a few parts of the tumor spheroidal surfaces were shed after treatment with Lf-MIONs/PFH; this shedding might result from the deformation of the cell skeleton. Comparison of the fluorescence intensity in the inner regions showed that the tumor spheroids treated by Lf$\mathrm{MIONs} / \mathrm{PFH}$ (Figure 5f) showed a stronger fluorescence distribution than that of the spheroids treated by Lf-MIONs (Figure 5e). The enlarged CLSM images of the in vitro penetration of particles into three-dimensional tumor spheroids provide a clear comparison of MIONs, Lf-MIONs, and Lf-MIONs/PFH (right images of Figure 5a, 5c, and 5e). To evaluate the rates of particle penetration, the fluorescence intensity was monitored and calculated at various times. Although both Lf-MIONs and Lf-PFH/MIONs at the beginning of $2 \mathrm{~h}$ 
display a rapid increase in fluorescence intensity, the Lf-PFH/MIONs have approximately double the intensity of Lf-MIONs after $2 \mathrm{~h}$ of incubation (Figure $\mathbf{5 g}$ and $5 \mathbf{h})$. A control experiment where no particles were incubated with tumor spheroids confirmed that the red color was due to the QD-labeled particles (Supplementary Material: Figure S10). Two possible mechanisms for the strong appearance of fluorescence at the inner regions of tumor spheroids can be sug-
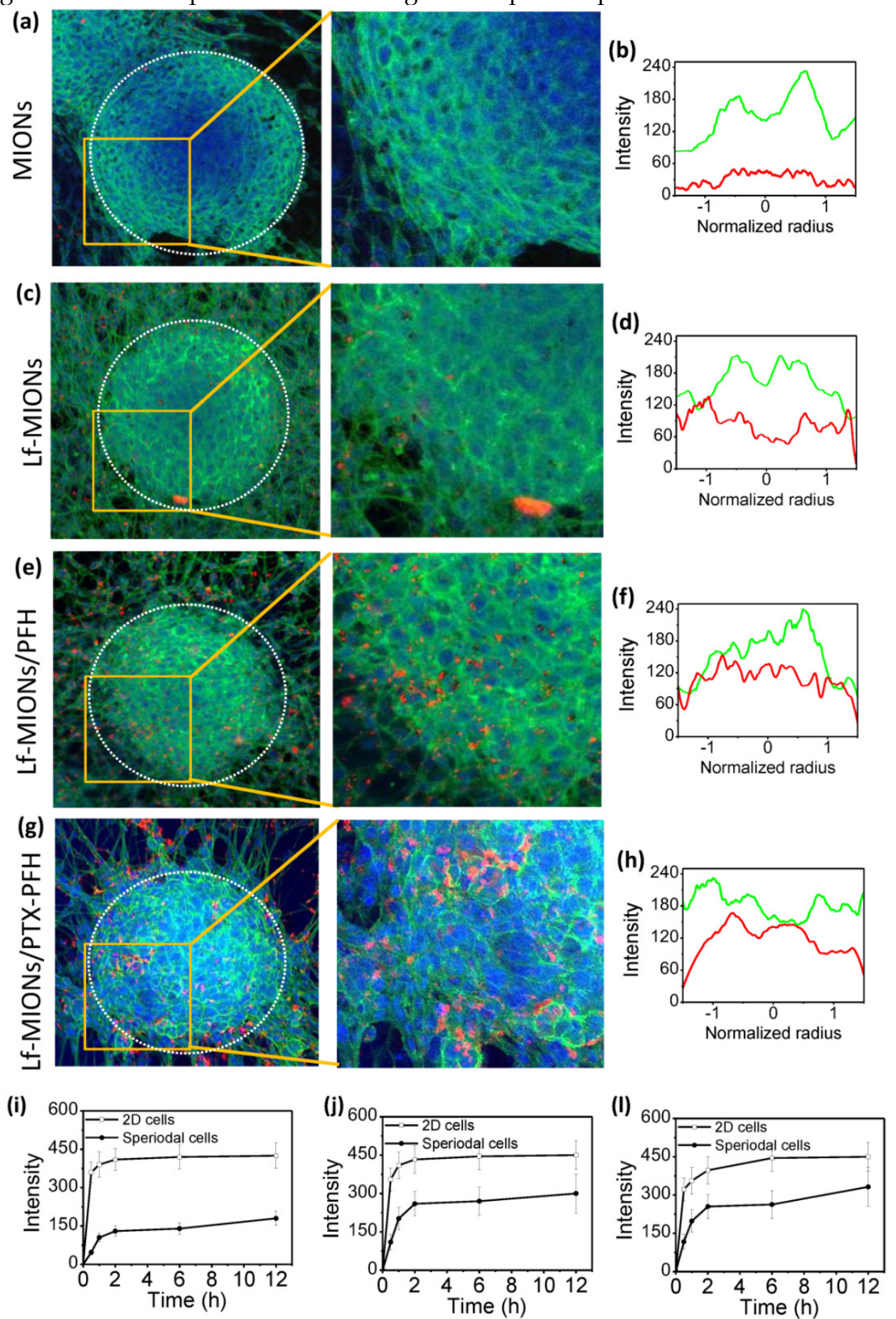

Figure 5. In vitro penetration of (a, b) MIONs, (c, d) Lf-MIONs, (e, f) Lf-MIONs/PFH and, (g, h) Lf-MIONs/PTX-PFH into three-dimensional tumor spheroids after 2 h of incubation. Dotted circles indicate the tumor spheroidal edges. Fluorescence intensity profiles of (b) MIONs, (d) Lf-MIONs, Lf-MIONs/PFH, and (h) Lf-MIONs/PTX-PFH in tumor spheroids. The green line is the cell skeleton; the red line is QD-labeled particles. (i-I) The fluorescence intensity of (i) Lf-MIONs, (j) Lf-MIONs/PFH, and (I) Lf-MIONs/PTX-PFH in the inner regions of tumor spheroids as a function of time. 


\section{MF effects on particle penetration}

To estimate the effect of MF on the particle penetration, an in vitro study was conducted on tumor spheroids incubated with Lf-MIONs/PFH for $4 \mathrm{~h}$, and then, the tumor spheroids were subjected to the MF for $1 \mathrm{~min}$. As shown in Figure 6a, a 3D-reconstructed cell image showed that large amounts of Lf-MIONs/PFH with bright red fluorescence appeared on the cells before the MF treatment. A closer observation revealed that parts of the particles could penetrate the inner regions and resided in the dense cell skeletons (Figure $\mathbf{6 b}$ and $\mathbf{6 c}$ ). The result showed that the tumor spheroids still maintained the morphology constructed by compact cells. In contrast, once the MF was applied for $1 \mathrm{~min}$, clear damage and deformation of the tumor spheroids were observed, as shown in Figure 6d. Parts of the boundary of the tumor spheroid seemed to be ruptured, and higher quantities of Lf-MIONs accumulated in the spheroid. In high-magnification CLSM images (Figure 6d and 6e), the cells exhibited shrinking morphologies, and clear gaps between cells could also be observed. The results indicated that the gasification of $\mathrm{PFH}$, which was triggered in a few seconds, significantly influenced the intercellular structures of tumor spheroids and destroyed the dense cell skeletons of compact cells in the periphery of the spheroids. This phenomenon also increased the particle penetration and accumulation in the tumor spheroids. As shown in Figure $6 \mathrm{~g}$, the particle fluorescence is approximately doubled in the inner regions of spheroids after the MF treatment for both Lf-MIONs and Lf-MIONs/PFH after another $4 \mathrm{~h}$ incubation. Notably, the MF also enhanced the accumulation of Lf-MIONs in tumor spheroids, which was probably cell damaged caused by the heat induced in a short time. For the time of accumulation, the fluorescence of the tumor spheroids that were incubated with Lf-MIONs/PFH rapidly increased within $1 \mathrm{~h}$ after the MF treatment; this response is much faster than that for the incubation with Lf-/MIONs (Figure 6h). This finding also implies that the Lf-MIONs/PFH caused severe harm to the tumor spheroids. This observation is considered to result from a combination of thermal and mechanical effects that increase the tumor micro-environmental permeability because the MF can induce the heat generation of MIONs and PFH gasification. Such effects also show the synergy of the carrier penetration enhancement and drug release in the tumor spheroids, which significantly increases the drug concentration in the deep regions of tumor cells.
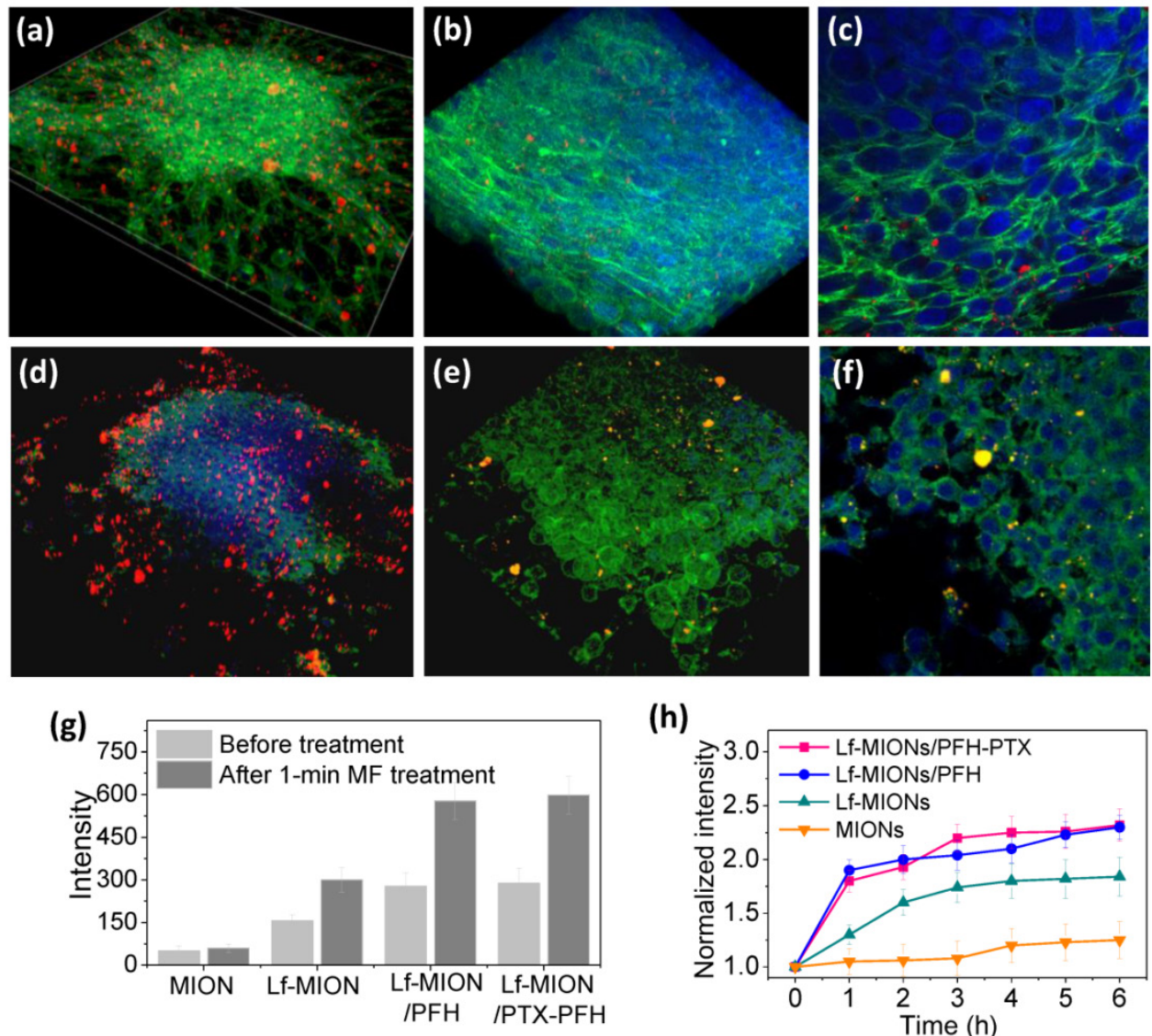

(h)

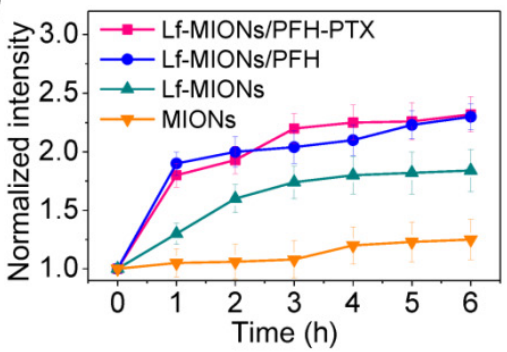

Figure 6. 3D-reconstructed cell image of tumor spheroids after incubation with Lf-MIONs/PFH (a-c) before and (d-f) after a 1-min treatment with a high-frequency magnetic field (MF). Lf-MIONs/PFH were labeled with bright red fluorescent QDs. (g) Fluorescence intensity of particles in the inner regions of tumor spheroids. (h) Normalized fluorescence intensity of particles as a function of time. 


\section{MF-triggered PFH/PTX release for in vitro cell killing}

Concerning MF treatments in tumor spheroids, the in vitro cytotoxicity of PTX-loaded MIONs, Lf-MIONs and Lf-MIONs/PFH (termed as MIONs/PTX, Lf-MIONs/PTX, and Lf-MIONs/PFHPTX, respectively) against tumor spheroids was estimated with and without MF treatments (Figure 7a and $7 \mathbf{b}$ ). Compared to Lf-MIONs/PTX and Lf-MIONs/PFH-PTX, MIONs/PTX exhibited lower cytotoxicity toward RG2 cells. The viability of the RG2 cells was approximately $47 \%$ even when the MION concentration was $20 \mu \mathrm{g} / \mathrm{ml}$. In contrast, Lf-MIONs/PTX and Lf-MIONs/PFH-PTX displayed enhanced cytotoxicity as the concentration increased. Consistent with the cell uptake experiments, the results demonstrated that the particles having high cell uptake ability can effectively transport PTX into the cells, leading to lower cell viability. Figure $7 \mathbf{b}$ shows the results of the cell-killing experiments using MF treatments at $4 \mathrm{kA} / \mathrm{m}$ of strength with various particles for $5 \mathrm{~min}$. Before being subjected to MF, the free particles in the incubation medium were removed to ensure that the particles were uptaken by the cells. Several conclusions can be obtained from these results. First, MF increases cytotoxicity when cancer cells are treated by MIONs/PTX, leading to $\sim 25 \%$ cell viability. This change is likely a consequence of MF-induced hyperthermia and PTX release via magnetic induction of MIONs although only a few MIONs were uptaken by cells. Second, the cell-killing efficacy of PTX-containing MIONs is enhanced by the addition of Lf, which lowers the cell viability to $15 \%$ at an Lf-MION concentration of $20 \mu \mathrm{g} / \mathrm{mL}$. Third, PFH -loaded Lf-MIONs also cause $\sim 20 \%$ cell viability via magnetic induction of MIONs, which achieves the high cell-killing efficiency owing to the combination of $\mathrm{PFH}$ gasifying and hyperthermia. Since the MF-induced PFH gasification, the gas in or on the cells can damage the cancer cell membranes, leading to cell death. For a similar concentration, the MF treatment for the combined PFH and PTX in the Lf-MIONs is highly efficient, lowering the cell viability to merely $4 \%$. Importantly, at the relatively low concentration, i.e., $5 \mu \mathrm{g} / \mathrm{mL}$, the Lf-MIONs/PTX can also significantly improve therapeutic effects compared to that for the particles without PFH. These results clearly agree and show that with the aid of a targeting ligand and $\mathrm{PFH}$, the MF-triggered approach that is made feasible by the MION formulation is successful for in vitro tumor spheroids; the cell-killing efficacy is considerably enhanced when any single element in this strategy (Lf, PFH, and MF treatment) is added to the treatment protocol.
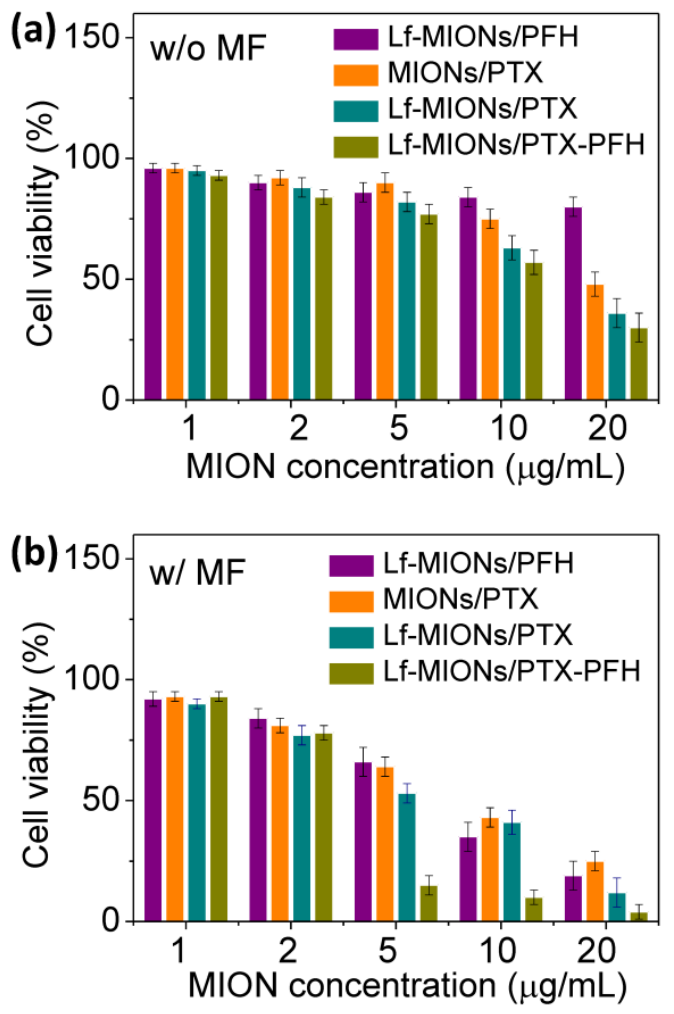

Figure 7. In vitro cell viability of MIONs/PTX, Lf-MIONs/PTX, and Lf-MIONs/PTX-PFH (a) before and (b) after 5-min of MF treatment.

\section{In vivo tumor therapy}

To investigate the tumor localization ability of Lf-MIONs/PFH, we injected $100 \mu \mathrm{L}$ of solution containing $0.5 \mathrm{wt} \%$ Lf-MIONs/PFH labeled with Cy 5.5 into nude mice bearing RG2 tumor cells via the tail vein. At a post-injection time of $24 \mathrm{~h}$, the MF was applied to the tumor for $5 \mathrm{~min}$. In Figure 8a, the fluorescent signal of Cy 5.5 (excitation wavelength: 640 $\mathrm{nm}$; emission wavelength: $710 \mathrm{~nm}$ ) was observed at the tumor site after three days both with and without MF treatments. However, the fluorescent intensity of the tumor was much stronger after the MF treatment than without MF, indicating the effective localization of the Lf-MIONs to the tumor cells. Such localization behavior is probably caused by the Lf on MIONs and the PFH gasification, which can increase the accumulation at RG2 cells and enhance the uptake efficiency, as we demonstrated in the in vitro study. Next, for evaluation of the antitumor activity, model mice with an RG2 tumor xenograft received the following treatments by i.v. injection via tail veins: (i) saline, (ii) MIONs/PTX, (iii) Lf-MIONs/PTX, and (iv) Lf-MIONs/PTX-PFH. At $24 \mathrm{~h}$ post injection, a $10 \mathrm{~min}$ MF treatment was applied to the tumor. As shown in Figure 8b, only treatment (iv) inhibited the increase in the tumor size, although treatments (ii) and (iii) arrested the tumor growth for the first 8 days. The results completely confirmed the therapeutic effects of 
the magneto-chemo-thermal therapy (i.e. Lf-MIONs/PTX) mediated by the MIONs in the carriers in the present study; however, the combined use of $\mathrm{MF}$, drug and $\mathrm{PFH}$ is the most effective, indicating that the PFH gasification in the tumor may help the particles penetrate, leading to an effective combination therapy in the solid tumor. The tumor growth inhibition rate of Lf-MIONs/PTX-PFH was approximately $90 \%$ compared with $78 \%$ for Lf-MIONs/PTX and $36 \%$ for MIONs/PTX (Figure 8c). Meanwhile, the mouse weight loss did not vary, demonstrating the minimal toxicity and side effects of the magneto-thermo-chemo-therapy administered using the Lf-MION/PTX-PFH systems (Supplementary Material: Figure S11).

In Figure 8d, the clearance organs of the mice were histologically investigated after intravenous injection of Lf-MIONs and MF treatment. After H\&E staining, these tissue slices (heart, lung, liver, spleen and kidney) were observed 5 days post treatment, and they did not show severe damage, indicating the low toxicity of the Lf-MIONs/PTX-PFH treatment of these

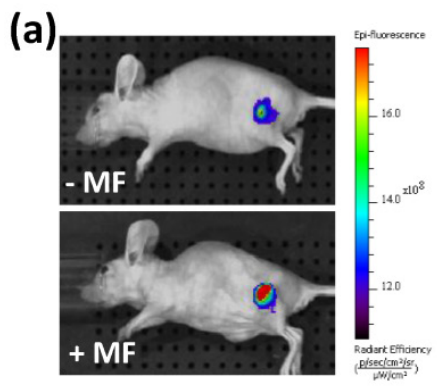

(b)

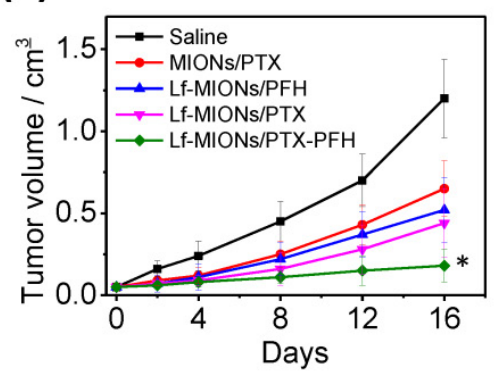

(c)
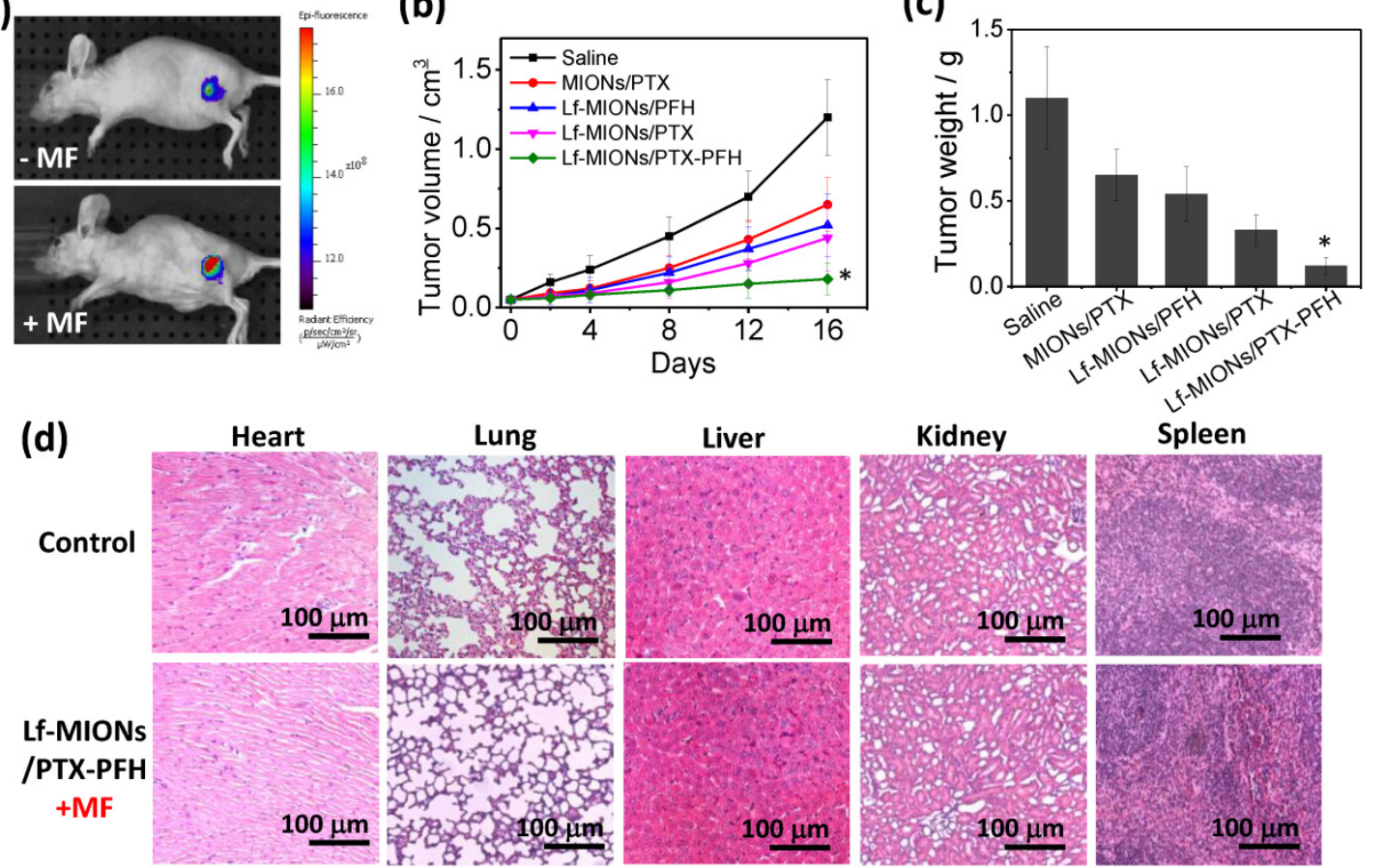

Spleen
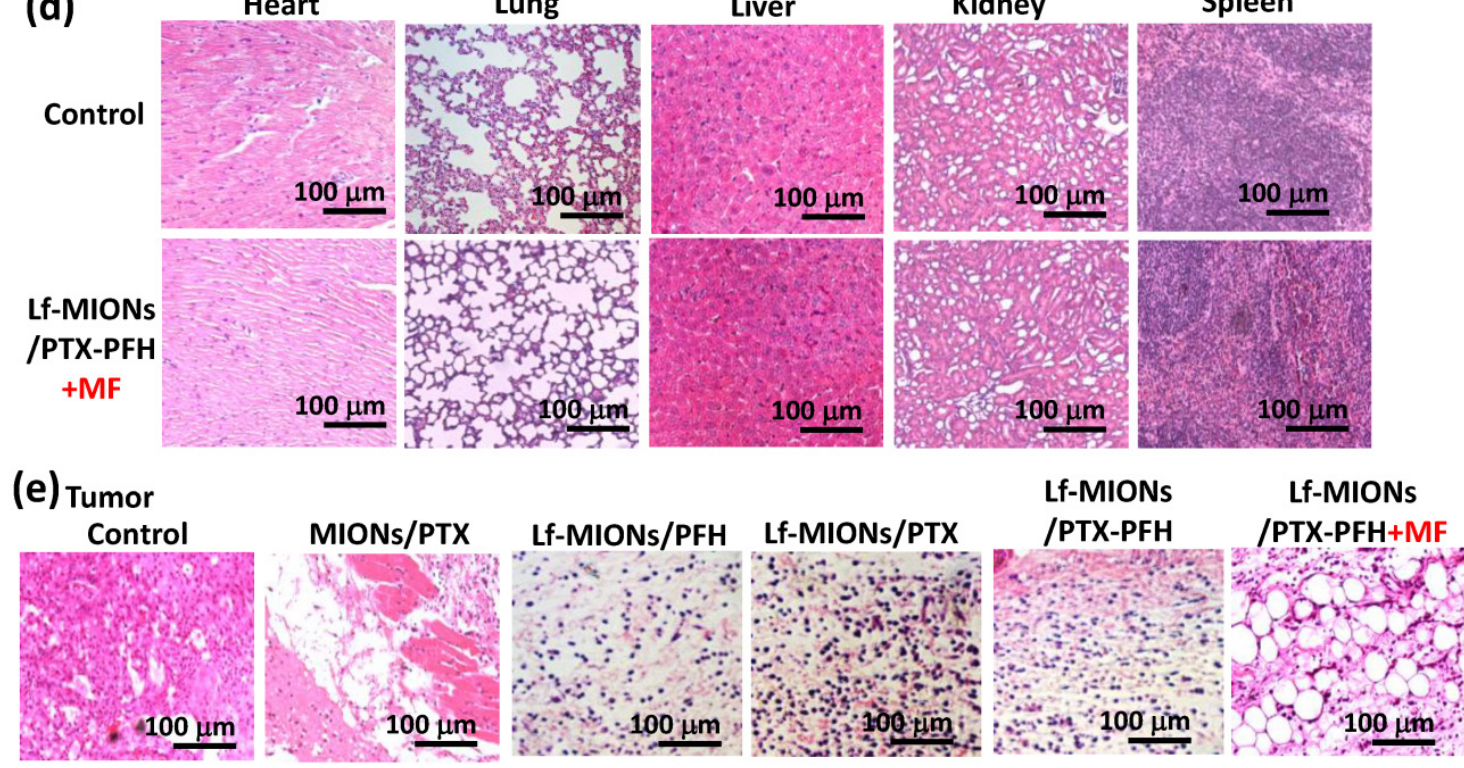

Figure 8. (a) Tumor accumulation of Lf-MIONs: fluorescence images of RG2 tumor-bearing mice following intravenous injection of Lf-MIONs with and without 10 min of MF treatment $24 \mathrm{~h}$ after injection. (b) Tumor growth curves of the RG2 tumor-bearing mice following intravenous injection of saline, MIONs/PTX, Lf-MIONs/PTX, Lf-MIONs/PFH, and Lf-MIONs/PTX-PFH. The MF was applied to the tumor for $10 \mathrm{~min} 24 \mathrm{~h}$ after injection. (c) Tumor weights of the RG2 tumor-bearing mice after the treatment at 16 days. (*) Statistically significant difference $\left({ }^{*} \mathrm{p}<0.05\right)$ as compared to all other groups. (d) H\&E-stained slices of clearance organs and RG2 tumors. The mice were sacrificed at 1 -day post-MF treatment. No obvious abnormality in major organs after the treatment. (e) Tumor slices after different treatments, including untreated (control), MIONs/PTX, Lf-MIONs/PTX, Lf-MIONs/PFH, Lf-MIONs/PTX-PFH, and Lf-MIONs/PTX-PFH+MF. 


\section{Conclusions}

In summary, we have developed a scalable and robust method to synthesize ultra-uniform mesoporous iron oxide nanoparticles (MIONs) with hydrophobic pores capped by lactoferrin (Lf) bio-gates. These particles were capable of encapsulating and delivering both hydrophobic PTX and PFH with high efficiency. Short-duration magneto-triggered PFH gasification was able to damage and rupture tumor spheroids, enhancing the penetration and accumulation of drug/carriers in deep tumors. A long-time MF treatment further led to intense heat and a burst-like drug release in tumor spheroids, causing high cell-killing efficiency of the thermo-chemo-therapy. The energy generation and drug release behaviors act rapidly and have a precise field-strength dependence without any lagging. From this combination of targeting and MF-guiding particle penetration strategies, considerable tumor growth suppression in vivo was also observed when treating a solid tumor. We believe that the drug delivery system developed here can promote the integration of theranostic approaches that combine bioimaging, controlled multiple-drug release, hyperthermia and deep tumor penetration for combination therapy and other biomedical applications.

\section{Supplementary Material}

Figures S1-S11, Table S1.

http://www.thno.org/v05p1233s1.pdf

\section{Acknowledgements}

This work was financially supported by the Ministry of Science and Technology of the Republic of China, Taiwan under Contract of MOST 103-2320-B-007-002-MY3 and MOST 103-2221-E-007-031-MY3, and National Tsing Hua University (104N2046E1 and 104N2733E1) in Taiwan.

\section{Competing Interests}

The authors have declared that no competing interest exists.

\section{References}

1. Barenholz Y. Doxil®--the first FDA-approved nano-drug: lessons learned. J Control Release. 2012; 160:117-34.

2. Montero AJ, Adams B, Diaz-Montero CM, Glück S. Nab-paclitaxel in the treatment of metastatic breast cancer: a comprehensive review. Expert Rev Clin Pharmacol. 2011; 4:329-34.

3. Angst MS1, Drover DR. Pharmacology of drugs formulated with DepoFoam: a sustained release drug delivery system for parenteral administration using multivesicular liposome technology. Clin Pharmacokinet. 2006; 45: 1153-76.

4. Dinndorf PA, Gootenberg J, Cohen MH, Keegan P, Pazdur R. FDA drug approval summary: pegaspargase (oncaspar) for the first-line treatment of children with acute lymphoblastic leukemia (ALL). Oncologist. 2007; 12: 991-8.

5. O'Brien ME1, Wigler N, Inbar M, Rosso R, Grischke E, Santoro A, Catane R, Kieback DG, Tomczak P, Ackland SP, Orlandi F, Mellars L, Alland L, Tendler C; CAELYX Breast Cancer Study Group. Reduced cardiotoxicity and comparable efficacy in a phase III trial of pegylated liposomal doxorubicin $\mathrm{HCl}$
(CAELYX/Doxil) versus conventional doxorubicin for first-line treatment of metastatic breast cancer. Ann Oncol. 2004; 15: 440-9.

6. Oerlemans C, Bult W, Bos M, Storm G, Nijsen JF, Hennink WE. Polymeric micelles in anticancer therapy: targeting, imaging and triggered release. Pharm Res. 2010; 27: 2569-89.

7. Xiao Y, Hong H, Matson VZ, Javadi A, Xu W, Yang Y, Zhang Y, Engle JW, Nickles RJ, Cai W, Steeber DA, Gong S. Gold Nanorods conjugated with doxorubicin and CRGD for combined anticancer drug delivery and PET imaging. Theranostics. 2012; 2: 757-68.

8. Hayashi K, Nakamura M, Miki H, Ozaki S, Abe M, Matsumoto T, Sakamoto W, Yogo T, Ishimura K. Magnetically responsive smart nanoparticles for cancer treatment with a combination of magnetic hyperthermia and remote-control drug release. Theranostics. 2014; 4: 834-44.

9. Liu HL, Fan $\mathrm{CH}$, Ting $\mathrm{CY}$, Yeh CK. Combining microbubbles and ultrasound for drug delivery to brain tumors: current progress and overview. Theranostics. 2014; 4: 432-44.

10. Hu SH, Chen SY, Gao X. Multifunctional nanocapsules for simultaneous encapsulation of hydrophilic and hydrophobic compounds and on-demand release. ACS Nano. 2012; 6: 2558-65.

11. Lai J, Shah BP, Zhang Y, Yang L, Lee KB. Real-Time Monitoring of ATP-Responsive Drug Release Using Mesoporous-Silica-Coated Multicolor Upconversion Nanoparticles. ACS Nano. 2015; 9: 5234-45.

12. Kobayashi H, Watanabe R, Choyke PL. Improving conventional enhanced permeability and retention (EPR) effects; what is the appropriate target? Theranostics. 2013; 4: 81-9.

13. Gradishar WJ, Tjulandin S, Davidson N, Shaw H, Desai N, Bhar P, Hawkins $\mathrm{M}$, O'Shaughnessy J. Phase III trial of nanoparticle albumin-bound paclitaxel compared with polyethylated castor oil-based paclitaxel in women with breast cancer. J Clin Oncol. 2005; 23: 7794-803.

14. Liu Z, Wang F, Chen X. Integrin targeted delivery of radiotherapeutics. Theranostics. 2011; 1: 201-10.

15. Heldin $\mathrm{CH}$, Rubin K, Pietras K, Ostman A. High interstitial fluid pressure - an obstacle in cancer therapy. Nat Rev Cancer. 2004; 4: 806-13.

16. Xiang D, Shigdar S, Qiao G, Wang T, Kouzani AZ, Zhou SF, Kong L, Li Y, Pu C, Duan W. Nucleic acid aptamer-guided cancer therapeutics and diagnostics: the next generation of cancer medicine. Theranostics. 2015; 5: 23-42.

17. Carmeliet P1, Jain RK. Principles and mechanisms of vessel normalization for cancer and other angiogenic diseases. Nat Rev Drug Discov. 2011; 10: 417-27.

18. Lu P, Weaver VM, Werb Z. The extracellular matrix: a dynamic niche in cancer progression. J Cell Biol. 2012; 196: 395-406.

19. Erez N, Truitt M, Olson P, Arron ST, Hanahan D. Cancer-Associated Fibroblasts Are Activated in Incipient Neoplasia to Orchestrate Tumor-Promoting Inflammation in an NF-kappaB-Dependent Manner. Cancer Cell. 2010; 17: 135-47.

20. Kalluri R, Zeisberg M. Fibroblasts in cancer. Nat Rev Cancer. 2006; 6: 392-401.

21. Ostman A1, Augsten M. Cancer-associated fibroblasts and tumor growth--bystanders turning into key players. Curr Opin Genet Dev. 2009; 19: 67-73.

22. Visvader JE, Lindeman GJ. Cancer stem cells in solid tumours: accumulating evidence and unresolved questions. Nat Rev Cancer. 2008; 8: 755-68.

23. Dean M, Fojo T, Bates S. Tumour stem cells and drug resistance. Nat Rev Cancer. 2005; 5: 275-84

24. Kim B, Han G, Toley BJ, Kim CK, Rotello VM, Forbes NS. Tuning payload delivery in tumour cylindroids using gold nanoparticles. Nat Nanotechnol. 2010; 5: 465-72.

25. Chauhan VP, Popović Z, Chen O, Cui J, Fukumura D, Bawendi MG, Jain RK. Fluorescent nanorods and nanospheres for real-time in vivo probing of nanoparticle shape-dependent tumor penetration. Angew Chem Int Ed Engl. 2011: 50: 11417-20

26. Huang K, Ma H, Liu J, Huo S, Kumar A, Wei T, Zhang X, Jin S, Gan Y, Wang PC, He S, Zhang X, Liang XJ. Size-dependent localization and penetration of ultrasmall gold nanoparticles in cancer cells, multicellular spheroids, and tumors in vivo. ACS Nano. 2012; 6: 4483-93.

27. Kumar A, Huo S, Zhang X, Liu J, Tan A, Li S, Jin S, Xue X, Zhao Y, Ji T, Han L, Liu H, Zhang X, Zhang J, Zou G, Wang T, Tang S, Liang XJ. Neuropilin-1-targeted gold nanoparticles enhance therapeutic efficacy of platinum(IV) drug for prostate cancer treatment. ACS Nano. 2014; 8: 4205-20.

28. Wong C, Stylianopoulos T, Cui J, Martin J, Chauhan VP, Jiang W, Popovic Z, Jain RK, Bawendi MG, Fukumura D. Multistage nanoparticle delivery system for deep penetration into tumor tissue. Proc Natl Acad Sci USA. 2011; 108 : 2426-31.

29. Jiang W, Kim BY, Rutka JT, Chan WC. Nanoparticle-mediated cellular response is size-dependent. Nat Nanotechnol. 2008; 3: 145-50.

30. Ta T, Porter TM. Thermosensitive liposomes for localized delivery and triggered release of chemotherapy. J Control Release. 2013; 169: 112-25.

31. Dicheva BM, ten Hagen TL, Schipper D, Seynhaeve AL, van Rhoon GC, Eggermont AM, Koning GA. Targeted and heat-triggered doxorubicin delivery to tumors by dual targeted cationic thermosensitive liposomes. J Control Release. 2014; 195: 37-48.

32. Watson KD, Lai CY, Qin S, Kruse DE, Lin YC, Seo JW, Cardiff RD, Mahakian LM, Beegle J, Ingham ES, Curry FR, Reed RK, Ferrara KW. Ultrasound increases nanoparticle delivery by reducing intratumoral pressure and increasing transport in epithelial and epithelial-mesenchymal transition tumors. Cancer Res. 2012; 72: 1485-93. 
33. Lai CY, Fite BZ, Ferrara KW. Ultrasonic enhancement of drug penetration in solid tumors. Front Oncol. 2013; 3: 204.

34. Chen YW, Chen PJ, Hu SH, Chen IW, Chen SY. NIR-triggered synergic photo-chemothermal therapy delivered by reduced graphene oxide/carbon/mesoporous silica nanocookies. Adv Funct Mater. 2014; 24: 451-459.

35. Pang Z, Feng L, Hua R, Chen J, Gao H, Pan S, Jiang X, Zhang P. Lactoferrin-conjugated biodegradable polymersome holding doxorubicin and tetrandrine for chemotherapy of glioma rats. Mol Pharm. 2010; 7: 1995-2005.

36. Wu C, Li F, Niu G, Chen X. PET imaging of inflammation biomarkers. Theranostics. 2013; 3:448-66.

37. Fang JH, Lee $\mathrm{YT}$, Chiang $\mathrm{WH}, \mathrm{Hu} \mathrm{SH}$. Magnetoresponsive virus-mimetic nanocapsules with dual heat-triggered sequential-infected multiple drug-delivery approach for combinatorial tumor therapy. Small. 2015; 11:2417-28.

38. Hu SH, Liao BJ, Chiang CS, Chen PJ, Chen IW, Chen SY. Core-shell nanocapsules stabilized by single-component polymer and nanoparticles for magneto-chemotherapy/hyperthermia with multiple drugs. Adv Mater. 2012; 24: $3627-32$

39. Fang Y, Zheng G, Yang J, Tang H, Zhang Y, Kong B, Lv Y, Xu C, Asiri AM, Zi J, Zhang F, Zhao D. Dual-pore mesoporous carbon@silica composite core-shell nanospheres for multidrug delivery. Angew Chem Int Ed Engl. 2014; 53: 5366-70.

40. Leong DT, Ng KW. Probing the relevance of 3D cancer models in nanomedicine research. Adv Drug Deliv Rev. 2014; 79-80: 95-106.

41. Albanese A, Lam AK, Sykes EA, Rocheleau JV, Chan WC. Tumour-on-a-chip provides an optical window into nanoparticle tissue transport. Nat Commun. 2013; 4: 2718.

42. Guo WM1, Loh XJ, Tan EY, Loo JS, Ho VH. Development of a magnetic 3D spheroid platform with potential application for high-throughput drug screening. Mol Pharm. 2014; 11: 2182-9.

43. Goodman TT, Olive PL, and Pun SH. Increased nanoparticle penetration in collagenase-treated multicellular spheroids. Int. J. Nanomedicine 2007, 2:265-274 